\title{
LXI. Remarks on some points of the reasoning in the recent discussions on the theory of the aberration of light
}

\author{
Rev. Baden Powell M.A. F.R.S. F.G.S. F.R.A.S.
}

To cite this article: Rev. Baden Powell M.A. F.R.S. F.G.S. F.R.A.S. (1846) LXI. Remarks on some points of the reasoning in the recent discussions on the theory of the aberration of light, Philosophical Magazine Series 3, 29:196, 425-440, DOI: 10.1080/14786444608645540

To link to this article: http://dx.doi.org/10.1080/14786444608645540

曲 Published online: 30 Apr 2009.

Submit your article to this journal $₫$

ЏII Article views: 2

Q View related articles $\sqsubset$ 


\section{LONDON, EDINBURGH AND DUBLIN}

\section{PHILOSOPHICAL MAGAZINE}

A N D

JOURNAL OF SCIENCE.

\section{[THIRD SERIES.]}

\section{$D E C E M B E R \quad 184.6$.}

LXI. Remarlss on some Points of the Reasoning in the recent Discussions on the Theory of the Aberration of Light. By the Rev. Baden Powel., M.A., F.R.S., F.G.S., F.R.A.S., Suvilian Professor of Geometry in the University of Oxford*.

THE explanation of the phænomenon of the aberration of 1 light, as given in most of the established treatises on astronomy, seems for at least a long time past, to have been generally acquiesced in ; until at the meeling of the British Association, $184.5+$, the subject was stirred anew by the announcement of the investigations of Prof. Challis and Mr. Stokes, the discussion of which has been continued in so many numbers of the Philosophical Magazine (vols. xxvii. xxviii.), and which seemed to open a new epoch in the history of the question.

The first impressions taken, and the reports which obtain currency in such a case, are not always to be trusted. But we were given to understand that we had hitherto been all along under an entire mistake with regard to aberration; that all the existing interpretations of it were unsatisfactory; that it in fact remained to this day wholly unexplained; that all previous writers had failed in showing the modus operandi; and that (in the nomenclature of Dr. Whewell) "the appropriate idea" was wholly wanting, until now for the first time supplied. Some again understood it to have been asserted, that we were now to believe no object really to lie in the direction which the ray coming from it seemed to indicate; that all objects, terrestrial as well as celestial, are subject to aberration; and that the astronomer, referring the star to the cross wire of his telescope, sees the wire out of its true place and not the star.

* Communicated by the Author.

+ See Report, Sectional Proceedings, p. 9.

Phil. Mag. S. 3. Vol. 29. No. 196. Dec. $18+6$. 
Again, the question was mixed up with the undulatory theory. It seemed to be considered, at least by some, that $a$ lengthy and abstruse analytical investigation was now necessary for anything like a complete explanation of what our predecessors thought sufficiently made out by a short popular illustration, or at most a simple geometrical construction.

On some of these points the two very able mathematicians who first started the question were themselves at issue: and though some other points, at first involved in paradox and mystery, were in some degree cleared up in the con'se of the discussion, yet it cannot be denied that much still remained which called for further elucidation; and even yet it cannot be said that the minds of the scientific world in general have been brought to any agreement, or perhaps any such clear and definite view of the real state of the case as to be able to come to a final decision, or to render useless an attempt to divest the question of some of the ambiguity and difficulty in which it has been involved.

I trust then it will not be deemed superfluous if, at a period when the immediate controversy seems to have arrived at a termination, I offer a few remarks which may assist in forming a dispassionate judgement on the merits of the discussion; and in doing so I will premise that the chief point really at issue seems to me to lie rather in the general nature of the reasoning and the principles of philosophical logic involved, than in those details to which the controversialists have more immediately addressed themselves.

I will then first advert to the question respecting the existing explanations of the facts, and afterwards to that respecting theories of light.

If we look to past times, from the date of the discovery of aberration, there certainly appears to have prevailed some little hesitation and difference of opinion as to the precise mode of viewing and explaining it.

Bradley himself seems to have felt an anxiety to dwell on any circumstance capable of aiding the better conception of his idea. Indeed, in the manner of his whole discussion we may acknowledge the justice of a remark made by Prof. Rigraul* on one portion of it, viz. that it "conveys the strong impression of its being the result of an inquiry which was new to the writer of it; and shows an evident unwillingness to omit anything which might bear upon the sulject." Bradley's original idea $\dagger$ (as is well known) was suggested by the chance

* Prof. Rigaud's Memoir and Miscellaneous Works of Bradley, p. xxxiv. Oxford, 1832 .

+ Rigaud's Memoir, p. xxx. Thomson's History of Royal Society, 346 . 
observation that the vane at the mast-head took a direction intermediate to that of the wind and of the boat: and the analogy was obvious between the direction in which the wind seemed by this index to come to the boat in motion, and that in which the light from a star seems to come to the earth in motion; the illustration however is but vague and general. And in the explanations thus adopted generally, that there was still something wanting to give a firm grasp to the apprehension, appears clearly, as well from the loose and wavering conceptions betrayed in some of the speculations of that time connected with the subject, as in the partial endeavours made by others to fix the view of it more precisely.

It is perhaps a proof of the defective ideas prevalent on the subject, that on the occasion of the transit of Venus, 1769 , Bliss and Hornsby should both have calculated the effect of aberration as accelerating the phases of the transit; while Prof. Winthorp of Cambridge, United States*, contended by means of a detailed illustration (imagining balls fired at a ship in motion), that the effect ought to be that of retardation, in which he was supported by Dr. Pricet.

A question somewhat of the same kind was also debated among the French astronomers; Monnier, Bailly and Cassini, contending that in a transit of a planet it was only necessary to consider the aberration of the sun; while Lalande introduced the difference of the aberrations of the sun and planet: and the former principle was defended by M. Jeaurat, on the ground that the planet was dark, and its existence only shown by the light of the sun surrounding it $\ddagger$.

The elaborate discussion of Euler $\oint$ contributes little perhaps to the precise explanation. He treats the whole subject in a geometrical form; considering the various cases of a luminous body seen by an observer in motion later in time than the truth, even if the observer be at rest, and different in direction if one or both be in motion : but in constructing for the composition of motions he makes no reference to any further essential conditions.

Even at a much later period, Dr. Young observed $\|$, "some mistakes appear to have been made respecting this subject; they may be avoided by attending to one general principle; that is, when a body moves uniformly forwards, the relative situation of another body, whether quiescent or in motion, appears at all times to be such as it really was at the moment of the emission of the light of the second body; in other words,

* Philosophical Transactions, 1770, p. 359. † Ibid, p. 536.

$\ddagger$ Mlén. Acad. Paris, 1786, p. 572. \$Comment, Petrop. xi. 150. 1739.

il Natural Philosophy, ii. 294. 


\section{Prof. Powell on the Theory of the Aberration of Light.}

neglecting the changes in the earth's motion during the passage of light, the apparent place of the sun, or of any star or planet, is its true geocentric place for the instant at which the light was emitted by the sun or star, or reflected by the planet:"

This explanation appears to me, I must confess, itself involved-in no inconsiderable obscurity. It may however indicate the kind of notions which were commonly prevalent, and which called for such an answer; and at any rate, the precise point of view in which the distinguished author thus placed the subject, will be at once seen to be quite distinct from that taken in the recent in vestigations.

Other writers seem to have felt in like manner the defective notions which prevailed, and sought to aid the more correct conception by various analogies. Lalande* illustrated the case by supposing a carriage with a front window open while rain falls vertically; when at rest no rain will enter, but when in motion it will. Maupertuis referred to the instance of hitting a bird fying, by aiming at a point in advance of its true place; and others have imagined various like cases.

But these popular illustrations fail in conveying precisely the idea of which we are in quest. 'The light from the star after all comes directly to us in the position where the earth is for the instant of observation; and that it is not the same ray which would have come to us at a previous instant, though it is one parallel to it (or in the case of a nearer body, differing only by its parallax), is indeed evident, but does not explain the fact, that the point indicated by the telescope differs from the real place of the star; and where and how, it would still be asked, does the deviation take place? It is probable that the more discerning students would have been led to some answer to this question, from considering attentively the necessity which obviously exists, and indeed on which the whole turns, for referring the star to some fixed point, that is to a point fixed relatively to the observer, though really in motion with him and the earth, such as the cross wire of the telescope.

Now in some even of the earliest explanations offered, it is, I think, clear that at least a near approach to a more precise statement of the case, including this essential idea, is distinctly made. In Bradley's original papert it is worthy of notice that he specially introduces the consideration of the direction of the tube of the telescope, and the light passing along its axis, as essential to the conception of the case (p. 64.7). But besides this, in his lectures at Oxford (which commenced in

- Astron. \$2831.

+ Philosophical Transactions, 1728. 
1729), he employed a small model (still preserved among the apparatus of the experimental lecture-room), which consists of a board or ruler made to move parallel to itself, while, by means of the same motion, a mark on a thread moves parallel to the ruler over pulleys attached to the fixed part, and consequently describes the diagonal while the ruler moves along the base*.

That this contrivance may be spoken of as showing in general the apparent direction in which the light would enter the eye, is indeed obvious. But the question still remains, rohat light is it which thus seems to come out of its real direction? and it would seem impossible to consider Bradley's model without perceiving that the mark passing down the diagonal must represent the ray passing down the tube of the telescope, which is itself in motion with the observer; and it is essentially this light which is the medium of the deception, since by composition of motions the diagonal actually coincides with another direction in space, viz. the direct course of the ray from the star: or to recur to the case of the boat, what is it which corresponds to the misleading vane? the answer could only be-the telescope.

'The fuller discussions given in the most approved elementary treatises, in pointing more precisely to the difference between the real place of the star at the instant, and that shown

* As it may not be uninteresting to preserve some notice, even of the slightest ideas, of so great a man as Bradley, I subjoin a rough sketch exhibiting the essential part of the little instrument referred to above. The top and side of the box are omitted to give a view of the interior; and it will be seen at a glance that the axis $\left(a a_{l}\right)$, which at once carries the screw $(s)$ (working in the teeth ( $\left.q q_{1}\right)$ fixed to $b$ ), and on which the two ends of the thread $\left(t t_{i}\right)$ wind in opposite directions, will by the same motion cause that thread to move over the pulleys $\left(p p_{t}\right)$, and give the sliding-board $(b)$ a lateral motion. On its lower side (which is the part to be exhibited to the spectator) is drawn a parallelogram, and its diagonal divided into equal parts, so that the longitudinal motion of a mark upon the thread may be clearly traced upon the diagonal as the parallelogram moves laterally.

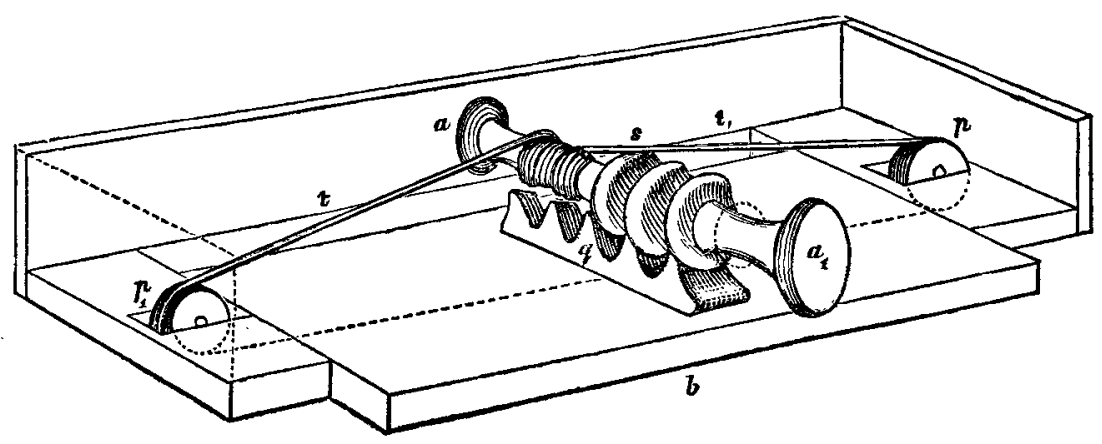


430 Prof. Powell on the Theory of the Aberration of Light.

by the direction of the telescope, manifestly implying that the light which comes down the telescope in motion coincides with that from the star at rest, it must be allowed, approach much nearer to a complete explanation, though they do not explicitly give it. It is true, several of these writers refer to speculations of another kind, which would maintain the same conclusion with regard to the naked eye, by supposing two impacts upon it, respectively proportional to the velocities of light and the earth, the resultant of which coincides with the direction of the ray. But they give such reasoning as entirely separate from the former: and Vince (p. 199, 8vo.) in particular censures those writers who would confound the two. Such reasonings are obviously insufficient as explanations of the aberration, as well from the uncertainty of the analogy assumed between the effects of light and those of mechanical impact, as on other grounds.

But by far the most lucid and satisfactory was the view of the subject taken by Clairaut*. In his memoir he gave perhaps the first complete investigation of the astronomical theory, leading to formulas for the aberration, not only in declination but in right ascension also, Bradley having originally considered only the former $t$ : and it is as the foundation of this investigation that he introduces his well-known illustration, so deservedly adopted by most subsequent writers, and which is peculiarly worthy of consideration in relation to our present object.

Fig. 1.

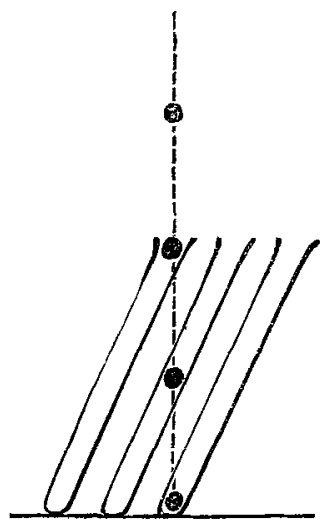

- Mem. Acad. Faris, 1737, p. 205.
Fig. 2 .

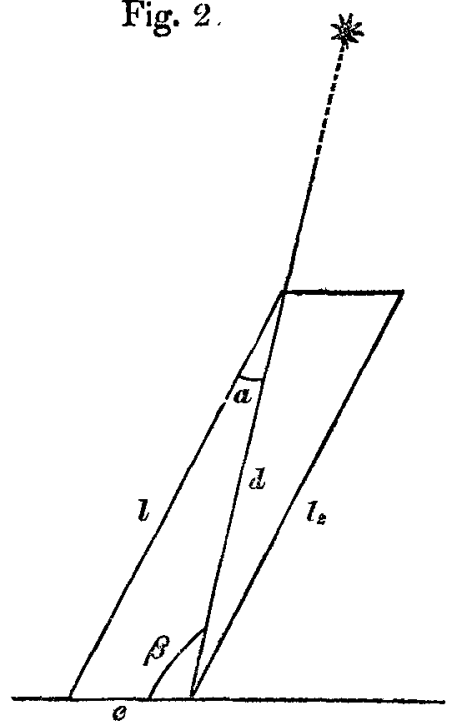

+ Rignud's Memoir, p. xxxiii. 
"In what direction must a tube be held by a person in rapid motion so as to catch at its bottom a drop of rain falling vertically?" (See fig. 1.) The answer to this question, if put into more geometrical language, would be simply the construction of a parallelogram whose diagonal is the vertical direction of the drop, and whose side and base are respectively proportional to the velocities of the drop and the tube, which consequently give its inclination; and the drop rohich was at the top of the tube at the beginning of the motion will be at the bottom of it at the end. It is but to translate this into the language of the actual case, to say that the light which comes down the tube of the telescope in the time in which the earth moves through the proportional space, will by composition of motions in the same time come down the vertical or diagonal, in which the light from the star comes directly, and with which it will thus coincide.

Clairaut, it is true, speaks explicitly only of the light from the star; but the essential reference to the tube, which he expressly points out ${ }^{*}$ as representing the direction of the telescope, is surely equivalent to the consideration of the light which comes along the telescope, or more precisely from its wire, to the eye. And in Sir J. Herschel's description of Clairaut's method + I conceive this is the interpretation implied; and hardly less distinctly, I think, in the same author's account of aberration in his treatise on Light $(\$ 10)$, as well as in the more elaborate discussion of Professor Woodhousef. Still that these writers fail in giving the idea its due prominence and full import, by explicit and formal statements, and the degree in which this affects the strict character of the investigation, will be best seen by a comparison with the professedly exact explanation as put forth by Prof. Challis.

That explanation, in the form in which it was stated in the course of the discussion referred to, was misunderstood; but it appeared to me that it readily admitted of being put under a slightly different form, by which means the essential principle (divested of all irrelevant and extraneous considerations with which it had been sometimes mixed up) might be at once rendered more perspicuous, and guarded against the possibility of misconception. As the shortest mode of stating it so as to obviate all objections, I would propose the following:-

1. Let $l$ and $e$ (see fig. 2) respectively be proportional to the known velocities of light, and of the earth in its orbit; let $d$ be the diagonal of a parallelogram of which $l$ and $e$ are

* Mém. Acal. Paris, 1737, p. 208.

$\uparrow$ Astrun. pp. 177, 178.

$\ddagger$ Ibid. i. 253 . 
432 Prof. Powell on the Theory of the Aberration of Lighi.

respectively the side and base; and let $\alpha \beta$ be respectively the angles which $d$ forms with $l$ and $e$; so that we have

$$
\frac{l}{e}=\frac{\sin \beta}{\sin \alpha}
$$

or if $\alpha$ be very small, $\quad \alpha=\frac{e}{l} \sin \beta$.

Also let $l_{2}$ be the side opposite and parallel to $l$.

2. Then conceiving $l l_{2}$ to be the successive positions of a telescope moving parallel to itself along with the earth throngh $e$, light coming from the top of $l$ to the observer's eye at the bottom, (or, more precisely, from some determinate point as the cross-wire,) relatively to the tube and the observer, will in the same time come down the diagonal $d$ relatively to space, by composition of motions.

3. If at the same time light from a star come directly in the direction $d$, this will coincide with the former; it will pass down the telescope as it moves, and the two objects will be referred by the eye to the same direction, and will appear to coincide thongh really separated by the angle $\alpha$, which is called the aberration.

4. Since the same inferences would apply also if another parallelogram be similarly constructed on the same diagonal, in like manner it will be seen that two objects moving with the observer would be referred to the same direction (in which they really are), though seen by light which really moves in the diagonal by composition of motions.

In this sense the aberration of terrestrial objects is spoken of; though it cannot be deternined by terrestrial observations.

5. In this investigation it is assumed that the light from the star comes in its original rectilinear course, and with the same velocity, equally whether the observer be at rest or in motion. It is also assumed that the velocity of light is that given by independent observations, and comparable in a known ratio with that of the earth in its orbit.

6. Thus, referring essentially to the light coming with the same velocity from the wire to the eye, it is shown that the aberration is explained by a vera causa; and fiom the $e x$ treme accuracy* with which the amount of aberration thus calculated agrees with that directly observed, it follows that the aberration is completely accounted for, and that there is no residual phonomenon. If there were any, then indeed recourse

* The mean of the direct observations, including the latest, given in Capt. Smyth's Cycle (ii. 401), is $\alpha=20^{t .43}$. The result of calculation, taking the velocity of light from observation of Jupiter's satellites at 8.2 $\min$. in traversing the rallius of the earth's orbit, gives $\alpha=20^{\prime \prime} \cdot 2$. 
must be had to other principles on which to find a complete explanation.

7. On this view the assumption of the rectilinear course is justified; and the explanation being complete, it is wholly superfluous to refer to the undulatory or any other theory of light, for an explanation of the phænomenon.

Upon this review of the principles of the explanation, it must, I think, be fully admitted that its most essential and characteristic feature is prominently brought out by Prof. Challis with far more distinct and systematic precision than by any of his predecessors in the inquiry : and from what has been remarked on their explanations, it will be judged to what extent we can consider them to have really implied the more precise principle, though they confessedly did not explicitly or systematically enunciate it. I may also here add, that perhaps it would render the matter still plainer to many apprehensions, if, instead of the telescope and its wire, we were to substitute the idea of a lamp elevated so as to appear against the sky to an observer below, and in coincidence with a star. Here then would be distinctly two rays; one from the star at rest, another from the lamp moving with the observer, apparently coinciding but really differing in direction.

There is perhaps one point on which it may be necessary to add a remark. In the above explanation it appears that the course of a ray propagated directly (as from the star) coincides with that given to another (propagated directly in a different course, as from the wire to the eye) by the motion of the object from which it originates along with that to which it comes; and it is hence inferred, that the two objects will be referred to the same direction as if the light from both were naturally propagated in the same direction. In other words, the course by which the star's light actually comes to the eye, relatively to the observer in motion, can be no other than the axis of the telescope, in which direction, the eye moving along with the light from the wire, at every instant receives an impression from it, along with one from the star; the one as truly comes down the tube as the other. But then, it may be asked, when we consider the very different modus operandi by which they each respectively take this direction, can we be sure that the effect ought to be the same?

This question may, I conceive, be answered by the consideration (quite independent of any theory of light), that as the light from the star comes down the tube only by virtue of the tube's motion, the whole effect of the ray still continues to be in its own direction; but the actual result must be estimated by resolving it, only that part of it which is in the direction of the tube being really effective on the eye. 


\section{Prof. Powell on the Theory of the Aberration of Light.}

But this question may appear to some perhaps superfluous; the above explanation, it may be said, only requires to have it shown that the two rays do actually assume the same direction in space by rohatever means; and this is all that is necessary to support the inference that the two objects must be referred to the same direction. Those who agree in this view will then merely consider any further discussion of this particular question as irrelevant to the complete explanation of the fact, though they may admit it as a question of curiosity.

Others however may not be satisfied without a further explanation; and whether that just offered be considered fully satisfactory or not, 1 confess it appears to me that some considerations of the kind are necessary for showing that the star and wire ought to be referred to the same direction.

The question raised as to sol at that direction is, also seems to be answered at the same time, if the foregoing view be admitted.

The impression produced is clearly that which results from the concurrence of two impressions, the one of the ray from the wire, the other of that from the star; the one simple, the other arising from its motion of propagation together with a motion of translation in the eye, but whose effect coincides. with the former: and a series of such compound impressions come in succession continuously in the direction of the axis of the tube to the eye. 'This circumstance appears to me decisive of the question, that both objects must be seen in the direction of the axis.

The explanation however was held to be independent of this question. It was said it would be equally valid should it be contended (as, for example, on any physiological or other grounds) that the eye received the impression not in the direction of the axis but in some other, as for instance that of the diagonal.

And when such a doubt was started, and when it was contended that the above explanation roas independent of any such question, or that the introduction of it was superfluous, a difficulty was raised in many minds; it was construed as if it tended to overset all the received notions as to the direction of vision, and to substitute something of an abstruse and theoretical kind; and the question raised, whether the star or the wire is seen displaced, led to serious objection, especially as coupled with the use of the term aberration applied to terrestrial objects.

But on the above view of the matter, the direction of vision does not appear an irrelevant consideration; and the question seems clearly answered. Thus I conceive we arrive at a precise statement of the case, and at the same time cut off a con- 
siderable source of cavil. The point determined by the position of the telescope and its wire is that to which the eye refers the star, which is consequently seen out of its true place by the amount of aberration, while the wire is seen in its true direction. The precise explanation then may be stated in all its essential particulars, divested of the mystery and obscurity which was sometimes found in it, and rendered at once simple, exact, and relieved of all perplexity from appenring at variance with received notions as to the direction of vision.

But a material part of the quetion before us relates to the investigation of the aberration in connexion with theories of the nature of light. A considerable portion of the existing controversy (we may observe) has arisen not only on the question of the details of such theoretical analysis, but also on the more general logical question of the grounds on which any such investigation should be undertaken at all.

As to the actual application of theory to the explanation of the phænomenon, Euler long since, adopting for simplicity the emission theory, admitted that some difference in the view taken of the case wonld result according to the hypothesis of light adopted*. He more particularly notices $(\$ 7)$ the objection which might arise on the emission theory, that light would partake in the velocity of the body from which it was projected, which would not be the case on the undulatory. This is further discussed $(\$ 24,25,28)$.

In the present state of the question it seems on all hands to be admitted that the point at issue is a very simple one. It in fact reduces itself ultimately to this: whatever theory we adopt, will it account for the uninterrupted maintenance of the law of the rectilinear propagation of light from a star to the earth in motion, at all parts of its course, but especially near the earth, with the same velocity?

The emission theory obviously fulfills this condition; as does also the undulatory, if the æther be at rest. But one of the greatest difficulties attending that theory, as formerly delivered, was the conception of the ather pervading all bodies and the interior of the earth yet remaining at rest, a passage being afforded to it through the interstices of the densest matter, in the words of Dr. Youngt, "as freely as the wind passes through a grove of trees," while the earth was in rapid mo-

* Comm. Petrop. xi. 150.

$\uparrow$ Phil. Trans., 1802, Experiments and Calculations on Physical Optics, $\$$ iv. It is remarkable that the author says he was led to this idea chiefly from considering the aberration of the stars. 
tion. This and other attendant objections, have at the present day led to the general admission of the æther being set in motion by the passage of the earth through it.

On this hypothesis the conclusion does not obviously or directly follow, but it may be shown to do so by an analytical investigation. That the theoretical investigations on the undulatory hypothesis, supposing the ather in motion, given both by Mr. Stokes and Prof. Challis, are of the highest value and importance, as evincing the capability of the undulatory theory to surmount the difficulty here presented, must be on all hands allowed, whatever may be thought of the points on which the two distinguished mathematicians differ.

The principle of $\mathrm{Mr}$. Stokes's investigation is, in a word, the analogy between sound and light; and the object is to show that the luminiferous waves are propagated through $a t h e r$ in motion, just as those of air constituting sound are propagated through a mass of air in motion, and that in both cases alike the impression reaches the organ just in the same manner as if the medium were at rest. Hence some limitations are introduced in the analysis; the very natural supposition that the æather near the earth acquires the same velocity as the earth, and thus remains, relatively to it, at rest, involving the condition that a certain expression $(u d x+v d y+w d z)$ is an exact differential.

Prof. Challis, on the other hand, discards these restrictions, allowing this expression to be an exact differential or not, and the motion of the æther any whatever: thus giving a greater degree of generality to the investigation.

But on the question whether we can legitimately infer that the motion acquired by the æther must be precisely equal to that of the earth, and whether, consequently, the more general assumption be necessary for a completely satisfactory conclusion,-or, on the other hand, whether the restrictions in the analysis are such as, without impairing its full applicability to the case, are more simple and natural, as well as on other minor points, I do not here enter: the main question with which we are at present concerned, is that which refers to the necessity for such theoretical investigation at all, as regards the complete explanation of the phænomenon: on which point also the two eminent disputants are at variance.

If what has been before advanced be assented to, I conceive while we deny the necessity for such an investigation as regards the explanation. of the facts, we must at least fully admit its importance as regards the credit of the theory.

We may illustrate the subject by taking a parallel case: granting the laws of reflexion and refraction, and the unequal 
refrangibility of light, the rainbow is perfectly explained without reference to the undulatory or any other theory of the nature of light. Yet it is material to the truth of that theory that it should explain the dispersion; and if it did so, as it confessedly explains the laws of reflexion and refraction, it would explain the rainbow, which is a mere consequence from them.

In other words, the aberration is not an independent property of light, like refraction or polarization, which require a theory to explain them, but the result (if the above principles be admitted) of simpler known causes. Any theory which explains these causes explains the aberration. But it is a matter of importance for the credit of any theory, that it should be able to explain them. Thus, more precisely, the question is not whether any theory explains the aberration, but whether it accounts for the facts from which the aberration is a consequence.

On the other hand, there are some, who, not entirely falling in with the above explanation, may view the whole matter under a different aspect, and may ask, before coming to any explanations, does not the naked fact of the aberration stand out as a prim $\hat{a}$ facie exception to the strict universality of the law of rectilinear propagation? and they may argue, as we are quite ignorant of the cause of the rectilinear motion of light, or the modus operandi which produces it, we cannot assume that as light approaches the earth in motion, it may not in some way be influenced, or a deviation caused : and it is only so far as we assume some theory of the nature of light that we can form any conception which may guide us to a conclusion on this point: and assuredly a theoretical investigation would in this point of view be not only desirable for the theory, but necessary for the explanation of the fact.

Those then who contend for the necessity for a theoretical investigation to explain the aberration, appear implicitly to assume that we cannot infer the absolute universality of the law of rectilinear propagation of light at all parts of its course to the earth in motion; though the result of the investigation on the undulatory hypothesis, with the æether in motion, is to establish it.

The question then is, whether this assumption be really called for in the nature of the reasoning: Is our ignorance of the nature and propagation of light so entire, that, for anything we know to the contrary, the mere circumstance of the rapid motion of the observer may in some unknown way act upon the ray of light as it approaches him and divert its course 


\subsection{Prof. Powell on the Theory of the Aberration of Light.}

\section{........" ten thcusand leagues away \\ Into the devious air"?}

or is such an idea so wholly improbable and unsupported by analogy that all consideration of it may be discarded, and the question rather put, What should jead us to imagine such an exception? or the onus probandi be thrown on the side of those who would assert it?

On this question I would only observe that every inductive law is essentially open to exceptions which may call for some modification of it, but any such exceptions must be substantiated on the most unequivocal evidence, before they can be admitted as influencing the universality of the law. If this be accepted as a fair rule of inductive logic, it will follow that we need not trouble ourselves with imagined or possible exceptions to the general law of rectilinear propagation, which may conceivably be occasioned by causes wholly imknown. If indeed we had any reasonable ground for suspecting that any known cause, such as $e$. g. terrestrial magnetism or electricity or heat, might act upon light as it approached the earth, then it would be a perfectly reasonable demand to see whether those causes produced any deviation, before we assumed the perfectly rectilinear course of the rays.

But without insisting on such considerations as those just referred to, it will I think be agreed on all hands, that, admitting the aberration to be wholly accounted for by the known facts relative to the motion of light and the earth, still the explanation cannot be called a perfect or philosophical one until those facts themselves are completely explained by a theory connecting them, and consequently the aberration, with the whole assemblage of laws and phænomena of light: and therefore that an appeal to the undulatory (or whatever is the bestestablished) theory is in this sense a necessary part of the investigation. Yet we should bear in mind that it is not more peculiarly essential in this case than in many others; such as e. $g$, the case of the rainbow before adverted to.

As another parallel case we might refer to the polarized rings. Granting the facts of polarization, and of the interferences of polarized light, the formation of the rings is completely explained without reference to any theory of the nature of light. It is a question for the credit of any theory, whether it will account for the laws of polarization and the interferences of polarized light: if it does so, it explains the rings; and the undulatory theory alone has been shown to do so. At the same time had we no theory explaining and connecting the laws of polarization and interference, we should certainly feel our views of the whole subject very unsatisfactory. 
In the case of aberration, the only difference perhaps is that the facts which explain the aberration are so much more simple and familiar that we feel satisfied almost without any theory of them at all.

Or again:- the retardation owing to light entering a denser medium, as the humours of the eye, and the question of its influence on the aberration, were discussed at an early period by Melvill (1753) and Wilson (1782) on the theory of emission and attraction; who for a long time did not perceive the compensation effected by the greater refraction*: this is in itself independent of theory. Yet the theoretical investigation was not unimportant.

Boscovich $\dagger$ also had maintained that an object viewed through a refracting medium in motion with the observer, will change its apparent place; and both he and Prof. Wilson had proposed to try it by means of a telescope filled with water. But Mr. Robison +, besides pointing out the inspracticability of this project, showed on theoretical grounds an oversight in the reasoning (though he had himself for a time embraced the idea), and concluded by establishing, on the emission theory, this proposition:- "If a ray of light moving in any direction, and with any velocity, meet with the surface of a refracting medium while it is in motion, its final relative motion will be the same as if the medium had been at rest, and the light had approached it with the same initial relative motion." ( $\mathrm{P} .106$. And questions somewhat akin to these have occupied the attention of more recent inquirers in connexion with the principles of undulations; and especially the conclusion that the laws of reflexion and refraction in general are uninfluenced by the motion of the ather along with the earth, has formed the subject of one portion of Mr. Stokes's investigations. And that such investigations are requisite for a complete and satisfactory theoretical view of the case, must on all hands be admitted.

Or to take a different illustration;--when the composition of forces and the experimental law of falling bodies were known, the explanation of the pendulum was complete, without any reference to the theory of universal gravitation. But it was incumbent on that theory to explain the law of falling bodies: and when it did so, it explained the pendulum. No one however doubts that it is highly valuable and satisfactory thus to connect the pendulum with universal gravitation. Here, indeed, the case is far stronger than in those of light, since we

* See Rigaud's Memoir of Bradley, p. xxxiii. and 483.

$\dagger$ Miscell. Works, vols. ii. and iv. 1785 . $\ddagger$ Ed. Trans. ii. 83, 1788. 
can here appeal to a vera causa of the highest and most comprehensive kind, whilst, however exactly the undulatory theory may explain any or all phænomena, it still does not carry us up to any vera causa, the existence of the æther having no independent proof, and being thus altogether hypothetical, as there is no more proof that the medium which resists Encke's comet is the same with the luminiferous æther, than there would be that atmospheric air is the same with inflammable gas.

LXII. Remarkable Solar Halos seen on the 19th of October 1846. By Edward Joseph Lowe*.

ON Monday, October 19, 1846, remarkable solar halos were observed at Highfield House, Nottinghamshire. The morning was fine with linear-cirri at a great altitude and cumuli floating beneath. The upper current drove the clouds from south, the lower from south-west.

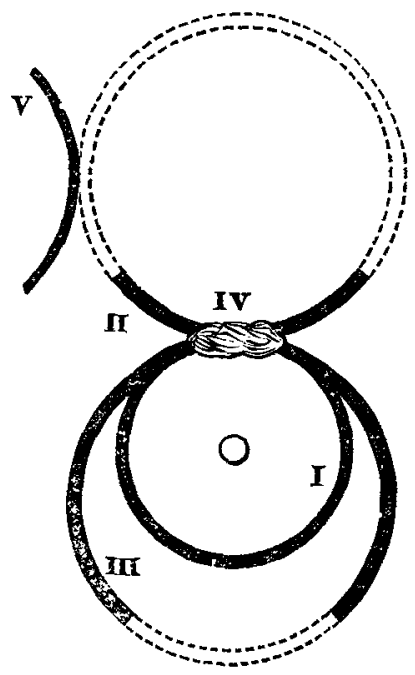

At $22^{\mathrm{h}}$ the phænomenon might besaid to have commenced, for the sky had assumed a hazy appearance, and a halo (I.) of $22^{\circ} 30^{\prime}$ radius had become visible: this was of a pale straw colour and soon became bright. The sky within the halo was considerably darker than that without.

$22^{\mathrm{h}} 10^{\mathrm{m}}$. An inverted arc of another halo (II.), of (apparently) $33^{\circ}$ radius, also of a pale straw colour, furmed at the vertex of the other halo, appeared.

At $22^{\mathrm{h}} 15^{\mathrm{m}}$ the halo (II.) had assumed a brilliant yellow colour, and the portion (IV.) which joined the halo (I.) a flame-like appearance, having widened considerably, being now about $3^{\circ}$ in width and $6^{\circ}$ in length. This brilliant parhelion, as I shall call it, became in a few minutes intense in brilliancy, vieing with the sun, for that luminary had been deprived of his usual brilliancy by haze, and had its rays carried out considerably. No change took place until $23^{\mathrm{h}} 30^{\mathrm{m}}$, when a third

* Communicated by the Author. 